\title{
Load-specific fiber reinforcements for aerospace applications made of inorganic fibers
}

\begin{abstract}
To reinforce high temperature materials, used in aerospace applications, ceramic fibers are the right material for a number of applications. To save weight, without losing performance, load-related fiber preforms with a near-net shape are suitable enhancement components. To produce this kind of customized preform, the brittle fiber material needs to be processed in a demanding textile process, which entails the risk of fiber damage. The article shows the influencing factors on fiber damage in a textile production process using inorganic brittle fiber materials through the example of weaving technology. The correlation between deflection angle, tensile stress and the choice of a suitable pattern is discussed with the aid of examples.
\end{abstract}

Keywords: ceramic fibers, reinforcement structures, composite materials, near-net shape, textile, load-specific
Volume 3 Issue 3 - 2019

\author{
Marielies Becker,' Frank Ficker,, ${ }^{1,2}$ Daniela \\ Schön' \\ 'Application Center for Textile Fiber Ceramics TFK at Fraunhofer- \\ Center for High Temperature Materials and Design HTL, Germany \\ ${ }^{2}$ Institute for Materials Science IFM, University Hof, Germany
}

Correspondence: Marielies Becker, Application Center for Textile Fiber Ceramics TFK at Fraunhofer-Center for High Temperature Materials and Design HTL, Kulmbacher Straße 76, 95213 Münchberg, Germany, Tel +49 928I 409-86I2,

Email marielies.becker@isc.fraunhofer.de

Received: August 14,2019 | Published: August 20, 2019

\section{Introduction}

In some application fields in aerospace applications, materials are exposed to short-term operating temperatures above $2000^{\circ} \mathrm{C}$ and at the same time significant temperature changes. ${ }^{1}$ Examples for these kind of areas are thermal protection systems like heat shields, thruster nozzles and turbine components. Fiber reinforced high temperature ceramics are suitable to meet these challenging material requirements. ${ }^{2}$ Compared to monolithic ceramics, fiber reinforced ceramics exhibit a high thermo-shock resistance, increased durability and non-catastrophic failure; they achieve high stiffness while having a low mass at the same time. ${ }^{3}$ Because of their excellent temperature resistance, ceramic fibers are suitable reinforcement components. ${ }^{1,2}$ Beside their high price, both fiber types exhibit brittle and fragile properties, which impede the technical production of complex textile preforms ${ }^{4}$ The sensitive and slow production is responsible for the limited availability of complex textile preforms in the market. A radical revision of the machine and processing concept is necessary to enable an economical production process. The Application Center for Textile Fiber Ceramics TFK at Fraunhofer-Center for High Temperature Materials and Design HTL develops and modifies textile production processes to make them suitable for the special requirements of ceramic fibers. Single and multilayer woven fabrics, braids and tape structures for the winding process have already been successfully implemented.

\section{Scientific investigation of thread guiding elements}

Within the framework of the project, different textile manufacturing methods have been monitored concerning their suitability for the processing of inorganic and in particular ceramic fibers. Because of their high flexibility in pattern design, the focus has been set on dobby and jacquard weaving technology. To save expensive fiber material and to avoid a high number of test-series, the individual mechanical stress has been analyzed for every machine part; especially the thread guiding elements have been in the focus of this investigation. To define the specific impact of every thread guiding element, different test setups have been developed. For the design of the test setups, the high fiber price has been taken into account. Therefore, the test equipment enables a comparison between different guiding elements with only very little material input. As significant influential factors, deflection radii, the profiles of the contact area and the surface quality of the thread guiding element have been taken under investigation.

For all tests, the tensile strength of the roving has been chosen as quantitatively measurable parameter to detect the degree of damage. All tensile tests have been based on DIN-EN 1007-5. In a first step, the intact roving has been measured to gain a comparable figure. To simulate the impact of different thread guiding elements, the prechosen elements can be inserted in the developed test setup. In this case, a number of different healds and eyelets have been compared to each other. Because of their high abrasion resistance, solely ceramic eyelets have been chosen for the investigation. In the test setup, the ceramic roving has been pulled through the thread guiding element. See Figures $1 \& 2$. The traction on the roving can be adjusted to simulate a load situation as similar to the selected weaving process as possible. Throughout the test, the roving has been chafed against the thread guiding element for 25 cycles.

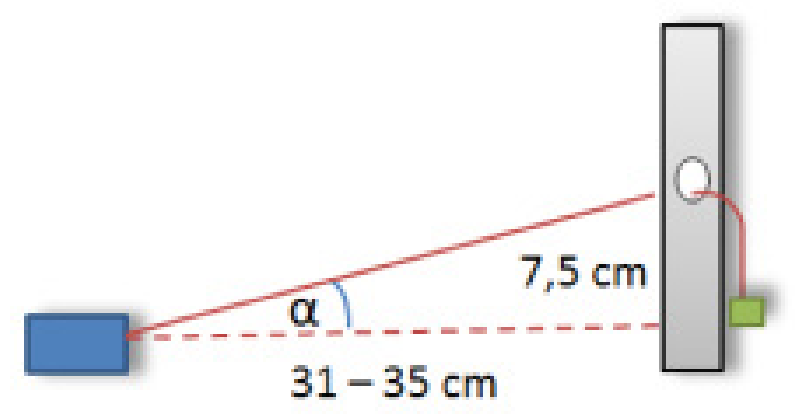

Figure I Schematic construction of thread leading element test. 


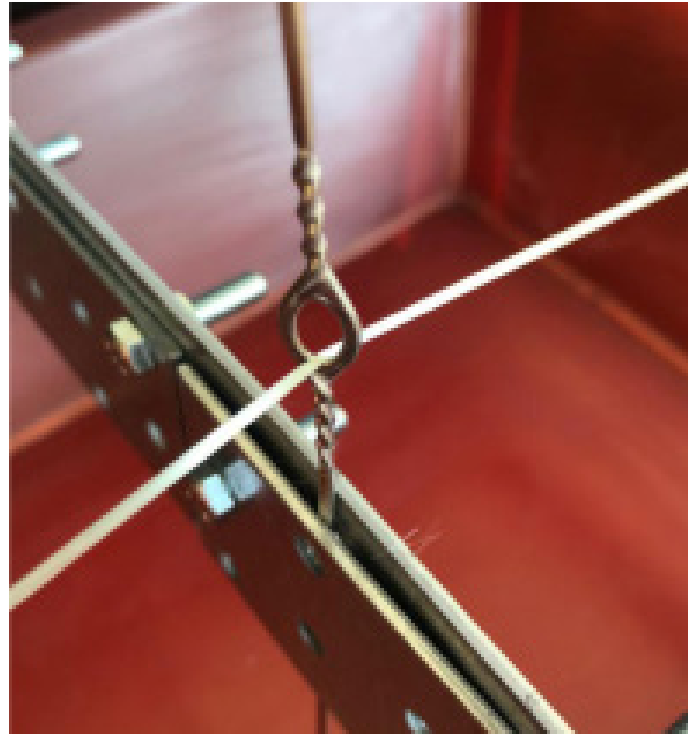

Figure 2 Ceramic fiber in heald.

To detect the impact of the deflection radius on the amount of fiber damage, a test design has been chosen, in which the diameter of the radius, the surface and the traction can be varied. Again, the roving is lead over the surface, and in this case, the test machine burdens the material cyclical. After all tests have been completed, the samples were rated visually with the aid of an optical microscope. Subsequently, the loss of strength caused by the tests has been measured and compared to the data of the intact roving. Results were interpreted relatively to each other.

\section{Textile processing of brittle fiber material}

Based on the data preserved through the earlier described tests, promising thread guiding elements have been defined for a further evaluation. The focus has been set on the practical implementation for dobby and jacquard weaving technology. These techniques have a high marked relevance and an extensive pattern variety, which make them especially suitable for custom-made structures. A dobby machine with a maximum of 240 warp threads has been chosen for a modification of all relevant guiding elements. To compare the test data with the technical condition and stresses during the weaving process, different textile processing series have been conducted. Starting from simple 2 dimensional fabrics, the complexity of the implemented structures has been continuously raised to detect technical limits of the material and the chosen machine set-up. Again, all fabrics have been evaluated concerning the degree of damage, using mechanical and optical analyses.

\section{Results}

The experiments clearly showed that the deflection radius and the tensile load have a significant impact on the amount of fiber damage during production. A small deflection radius stresses the fibers and causes fiber damage. The higher the brittleness of the fiber, the higher is the sensitivity against deflection in combination with a high tensile load. Silicon Carbide ( $\mathrm{SiC}$ ) fibers have for example been tested with deflection radii from $80-20 \mathrm{~mm}$. After 20 test cycles the samples which have been tested with a radius of $80 \mathrm{~mm}$ showed a strength reduction of $33 \%$ compared to the original roving strength. Samples that have been tested under same conditions but with a radius of $20 \mathrm{~mm}$ showed a strength reduction of $39 \%$. But not only a high tensile load showed an impact on the fiber breakage, but a very low tensile load also had a negative impact on the bundle. If the roving is pulled over a deviation point with very low tensile load, the roving material tends to separate. In this case, single filaments have a higher risk to tangle and break. Not surprisingly, the results of the mechanical tests showed that especially intermittent stress is critical for the roving. See Figure 3.

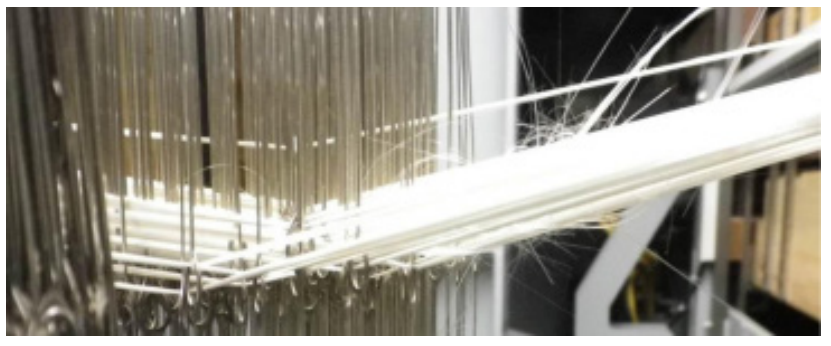

Figure 3 Fiber breakages at heald.

To reduce the intermitted stress, healds with a large radius, a big contact area and a smooth contact surface have been chosen. Concerning the tested eyelets, the versions with a polished surface showed a reduced fiber breakage compared to not polished surfaces. Further, the test results lead to the assumption that eyelets with a bigger contact area, caused by a longer design, have a positive effect on the processing properties. Summarizing, the results clearly show that even small contact areas have a big impact on the amount of fiber damage and need therefore to be chosen carefully. The thread leading elements, which showed the best processing properties with regards to brittle ceramic fibers, have been chosen for pursuing weaving tests. On top of the selection of suitable thread guiding elements, the weaving pattern needs to take the brittle fiber properties and the limited possibility of deflection angles into account. Starting from simple single layer woven fabrics, more complex structures have been implemented. To design load-specific reinforcing structures with a near-net-shaped design, the brittle fiber material does not only have to be processed in the correct way, the position of every fiber in the final textile structure needs to be considered as well. The preliminary tests showed that every fiber-type has a minimum deflection radius. Based on this knowledge, pattern designs have been developed in which the fiber material has enough space to swerve in the area of deflections and thus reduce the tension (Figures $4 \& 5$ ).

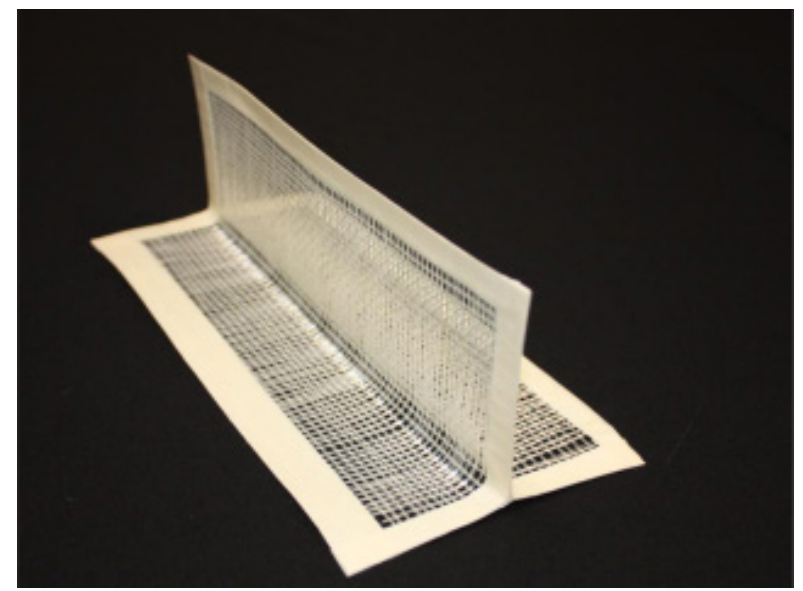

Figure 4 Seamlessly woven T-shaped preform made of $\mathrm{Al}_{2} \mathrm{O}_{3}$ rovings. 


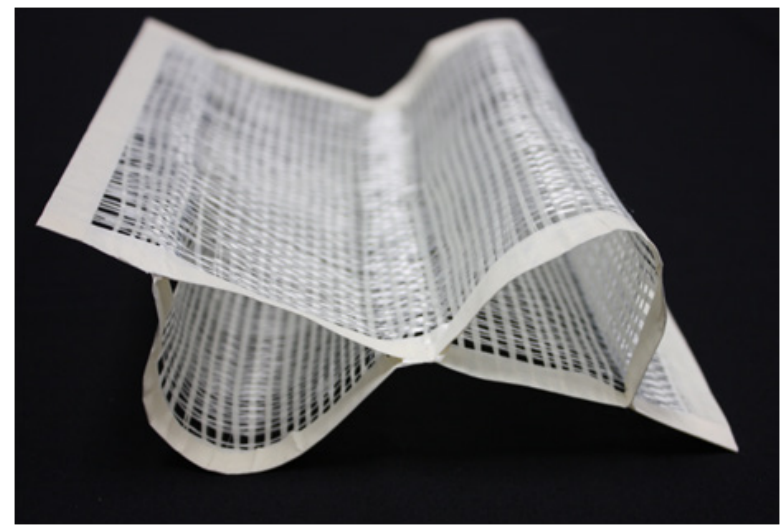

Figure 5 Seamlessly woven near net shaped preform made of $\mathrm{Al}_{2} \mathrm{O}_{3}$ roving. Discussion

The evaluation of the data clearly showed that first 3 dimensional woven structures have been implemented successfully, although not all influencing factors for fiber damage during the weaving process have been investigated. Only the damaging factors after unwinding the roving from the spool and before weaving have been taken under consideration. Even though the amount of fiber breakage of ceramic fibers has been reduced by choosing optimized thread guiding elements and an adjusted pattern design, damage factors such as accruing during weft insertion have been excluded. The here listed test results summarize technical statements which are universal for all brittle fiber materials, but intricacies can vary between different typesdepending on strength and stiffness of the ceramics.

\section{Conclusion}

The processing of brittle fiber materials to complex textile preforms can be technically implemented. To enable a stable and economic process, an extensive adaptation of the equipment is necessary. The adjustment includes the optimization of contact surfaces, contact geometries and fiber stress. The results although showed that the more sensitive a material is, the more carefully all machine elements need to be chosen. Therefore, the introduced tests need to be performed and analyzed for all different ceramic materials individually. If the marked and technical trend is moving towards load-specific fiber enhancement structures, not all production techniques are suitable. Also the physical limits of the fiber material exclude some preform geometries with small angles.

In the future, the development focus will be on custom-made textile structures, which will enable a targeted enhancement in all three dimensions and creates the possibility to reduce fiber input to a minimum. Due to high set-up costs and limited adjustment options, a fully automated production of small quantities still needs further machine and program development work.

\section{Funding details}

This research project was sponsored by Bayerisches Staatsministerium für Wirtschaft, Landesentwicklung und Energie

\section{Acknowledgments}

We would like to thank the University of Hof and especially Alexandra Luft, Corinna Anzer and Roxana Miksch for the cooperation in this project.

\section{Conflicts of interest}

Authors declare that there is no conflict of interest.

\section{References}

1. Tang S, Hu C. Design, Preparation and Properties of Carbon Fiber Reinforced Ultra-High Temperature Ceramic Composites for Aerospace Applications: A Review. Journal of Materials Science \& Technology. 2017;33(2):117-130.

2. Silicon Carbide (SiC) Fiber-Reinforced SiC Matrix Composites. National Aeronautics and Space Administration, Technology Transfer Office; 2015

3. Krenkel W. Ceramic Matrix Composites. Wiley-vch Verlag: Weinheim; 2008.

4. Bergmann HW. Konstruktionsgrundlage für Faserverbundbauteile. Springer-Verlag: Berlin; 1992. 